\title{
The Schrödinger functional with chirally rotated boundary conditions*
}

\author{
Stefan Sint ${ }^{\dagger}$ \\ Universidad Autónoma de Madrid \\ Instituto de Física Teórica CSIC/UAM \\ E-28049 Cantoblanco, Madrid, Spain \\ E-mail: stefan.sinteuam.es
}

\begin{abstract}
Using orbifold techniques I construct the Schrödinger functional (SF) for a doublet of Wilson quarks with chirally rotated boundary conditions. This allows to perform checks of universality: for instance, the renormalized SF coupling constant, defined with either boundary conditions, must have a unique continuum limit. Similarly, SF correlation functions in twisted mass QCD and standard QCD can be defined such that they share a common continuum limit. An additional benefit of the new set-up consists in the observation that all the bulk $\mathrm{O}(a)$ counterterms to the action and composite operators become irrelevant in the chiral limit. This implies that (ratios of) SF renormalization constants can be automatically $\mathrm{O}$ (a) improved, up to the effect of unavoidable boundary counterterms. As a first application we calculate the running coupling for $N_{\mathrm{f}}=2$ flavours in the SF-scheme to one-loop order of perturbation theory. Universality of the continuum limit is confirmed and the irrelevance of the Sheikholeslami-Wohlert term in the action is demonstrated explicitly.
\end{abstract}

XXIIIrd International Symposium on Lattice Field Theory

25-30 July 2005

Trinity College, Dublin, Ireland

*FTUAM-05-13 and IFT-UAM-CSIC/05-39

† Speaker. 


\section{Introduction}

The Schrödinger functional (SF) has become a general tool to address non-perturbative renormalization problems in $\mathrm{QCD}^{1}$. Renormalization schemes based on the SF are gauge invariant, quark mass independent (through renormalization in the chiral limit) and suitable for evaluation by both Monte Carlo and perturbative methods. Moreover, the finite space-time volume is used to set the renormalization scale, so that recursive finite size techniques become applicable.

Why should one be interested in changing the boundary conditions for the quark and antiquark fields? The first motivation comes from twisted mass QCD: as discussed in [4], correlation functions calculated in renormalized twisted mass QCD are, up to cutoff effects, related to renormalized correlation functions in standard QCD by a non-singlet chiral rotation. For this statement to hold true with SF correlation functions, the SF boundary conditions must be chirally rotated, too. Formulating the SF with the same boundary conditions for both twisted mass QCD and standard QCD (as was done in [5]) implies that the renormalised SF correlation functions are different even in the continuum limit. Second, it can be shown that, in a finite volume with (some variant of) periodic boundary conditions, $\mathrm{O}(a)$ improvement of massless Wilson quarks is automatic (cp. [6]). While the argument does not go through in the presence of standard SF boundary conditions, it can be resurrected in the chirally rotated set-up.

\section{Chiral rotation and SF boundary conditions}

Consider isospin doublets $\chi^{\prime}$ and $\bar{\chi}^{\prime}$ of quark and anti-quark fields satisfying homogeneous SF boundary conditions [7] $\left(P_{ \pm}=\frac{1}{2}\left(1 \pm \gamma_{0}\right)\right.$,

$$
\begin{array}{ll}
\left.P_{+} \chi^{\prime}(x)\right|_{x_{0}=0}=0, & \left.P_{-} \chi^{\prime}(x)\right|_{x_{0}=T}=0, \\
\left.\bar{\chi}^{\prime}(x) P_{-}\right|_{x_{0}=0}=0, & \left.\bar{\chi}^{\prime}(x) P_{+}\right|_{x_{0}=T}=0 .
\end{array}
$$

When performing a chiral field rotation,

$$
\chi^{\prime}=\exp \left(i \alpha \gamma_{5} \tau^{3} / 2\right) \chi, \quad \bar{\chi}^{\prime}=\bar{\chi} \exp \left(i \alpha \gamma_{5} \tau^{3} / 2\right),
$$

the rotated fields satisfy the chirally rotated boundary conditions

$$
\begin{aligned}
\left.P_{+}(\alpha) \chi(x)\right|_{x_{0}=0} & =0, & \left.P_{-}(\alpha) \chi(x)\right|_{x_{0}=T} & =0, \\
\left.\bar{\chi}(x) \gamma_{0} P_{-}(\alpha)\right|_{x_{0}=0} & =0, & \left.\bar{\chi}(x) \gamma_{0} P_{+}(\alpha)\right|_{x_{0}=T} & =0,
\end{aligned}
$$

with the projectors

$$
P_{ \pm}(\alpha)=\frac{1}{2}\left[1 \pm \gamma_{0} \exp \left(i \alpha \gamma_{5} \tau^{3}\right)\right] .
$$

For $\alpha=0$ the standard projectors $P_{ \pm}=P_{ \pm}(0)$ are recovered. The special case of $\alpha=\pi / 2$ will be of particular interest in the following,

$$
P_{ \pm}(\pi / 2) \equiv Q_{ \pm}=\frac{1}{2}\left(1 \pm i \gamma_{0} \gamma_{5} \tau^{3}\right),
$$

as the orbifold method cannot be applied for arbitrary values of $\alpha$.

\footnotetext{
${ }^{1}$ see $[1,2]$ for an overview and [3] for a recent application and further references.
} 


\section{Orbifold construction}

In the context of lattice QCD orbifold techniques have been applied by Taniguchi [8] in order to implement SF boundary conditions for Ginsparg-Wilson quarks. Here I consider a single Wilson quark flavour with lattice action

$$
S_{f}[\psi, \bar{\psi}, U]=a^{4} \sum_{-T<x_{0} \leq T} \sum_{\mathbf{x}} \bar{\psi}(x)\left(D_{W}+m_{0}\right) \psi(x) .
$$

The fermionic fields are taken to be $2 T$-anti-periodic in the Euclidean time direction,

$$
\psi\left(x_{0}+2 T, \mathbf{x}\right)=-\psi(x), \quad \bar{\psi}\left(x_{0}+2 T, \mathbf{x}\right)=-\bar{\psi}(x),
$$

and an orbifold reflection is introduced about the point $x_{0}=0$,

$$
R: \psi(x) \rightarrow i \gamma_{0} \gamma_{5} \psi\left(-x_{0}, \mathbf{x}\right), \quad \bar{\psi}(x) \rightarrow \bar{\psi}\left(-x_{0}, \mathbf{x}\right) i \gamma_{0} \gamma_{5}
$$

Following [8] the gauge field can be treated as an external field. It is first extended to the doubled time interval $[-T, T]$, through

$$
U_{k}\left(-x_{0}, \mathbf{x}\right)=U_{k}\left(x_{0}, \mathbf{x}\right), \quad U_{0}\left(-x_{0}-a, \mathbf{x}\right)^{\dagger}=U_{0}(x),
$$

and then $2 T$-periodically continued to all Euclidean times.

The fermionic fields may now be decomposed in even and odd components with respect to the reflection symmetry $R$, viz.

$$
R \psi_{ \pm}= \pm \psi_{ \pm}, \quad R \bar{\psi}_{ \pm}= \pm \bar{\psi}_{ \pm} .
$$

Even and odd fields then satisfy Dirichlet conditions at $x_{0}=0$,

$$
\left(1 \mp i \gamma_{0} \gamma_{5}\right) \psi_{ \pm}(0, \mathbf{x})=0, \quad \bar{\psi}_{ \pm}(0, \mathbf{x})\left(1 \mp i \gamma_{0} \gamma_{5}\right)=0 .
$$

Due to the $2 T$-anti-periodicity the complementary components then satisfy Dirichlet conditions at $x_{0}=T$ :

$$
\left(1 \pm i \gamma_{0} \gamma_{5}\right) \psi_{ \pm}(T, \mathbf{x})=0, \quad \bar{\psi}_{ \pm}(T, \mathbf{x})\left(1 \pm i \gamma_{0} \gamma_{5}\right)=0 .
$$

For the orbifold projection to work, a consistency condition must be satisfied,

$$
S_{f}[\psi, \bar{\psi}, U]=S_{f}\left[\psi_{+}+\psi_{-}, \bar{\psi}_{+}+\bar{\psi}_{-}, U\right]=S_{f}\left[\psi_{+}, \bar{\psi}_{+}, U\right]+S_{f}\left[\psi_{-}, \bar{\psi}_{-}, U\right] .
$$

This condition is rather strong and is the reason why the construction is restricted to the choice $\alpha=\pi / 2$. As a consequence, the functional integral over the fermion fields factorises. Interpreting $R$-even and $R$-odd fields as flavour components of a doublet field,

$$
\chi=\sqrt{2}\left(\begin{array}{l}
\psi_{-} \\
\psi_{+}
\end{array}\right), \quad \bar{\chi}=\sqrt{2}\left(\bar{\psi}_{-}, \bar{\psi}_{+}\right),
$$

the functional integral over the quark fields takes the form

$$
\int \prod_{-T<x_{0} \leq T} \mathrm{~d} \psi(x) \mathrm{d} \bar{\psi}(x) \mathrm{e}^{-S_{f}[\psi, \bar{\psi}, U]} \propto \int \prod_{0 \leq x_{0} \leq T} \mathrm{~d} \chi(x) \mathrm{d} \bar{\chi}(x) \mathrm{e}^{-\frac{1}{2} S_{f}[\chi, \bar{\chi}, U]} .
$$


The fields in the orbifolded theory satisfy the boundary conditions

$$
\begin{array}{ll}
\left.Q_{+} \chi(x)\right|_{x_{0}=0}=0, & \left.Q_{-} \chi(x)\right|_{x_{0}=T}=0, \\
\left.\bar{\chi}(x) Q_{+}\right|_{x_{0}=0}=0, & \left.\bar{\chi}(x) Q_{-}\right|_{x_{0}=T}=0,
\end{array}
$$

which are a special case of Eqs. (2.3). Furthermore, in Eq. (3.10) we have used the fact that the independent field variables $\chi$ and $\bar{\chi}$ may be taken to be the fields at Euclidean times $0<x_{0}<T$ and the non-Dirichlet components at $x_{0}=0, T$. Finally, a complete reduction to the interval $[0, T]$ is obtained by re-writing the lattice action

$$
S_{f}[\chi, \bar{\chi}, U]=2 a^{4} \sum_{0 \leq x_{0} \leq T} \sum_{\mathbf{x}} \bar{\chi}(x) \mathscr{D} \chi(x) .
$$

Here $\mathscr{D}$ is essentially the Wilson-Dirac operator including the standard bare mass term, except for some modifications near the time boundaries which are induced by the orbifold reflection. The factor of 2 originates from the fact that the contributions from negative and positive times are equal, and has been anticipated in Eq. (3.9). The explicit form of $\mathscr{D}$ and the identification of the dynamical field space are the main results of the orbifold construction which would have been difficult to obtain in other ways.

\section{Boundary counterterms}

Using the lattice symmetries of the chirally rotated SF the possible boundary counterterms of dimension 3 can be identified:

$$
K_{1}=\bar{\chi} \gamma_{5} \tau^{3} \chi, \quad K_{ \pm}=\bar{\chi} Q_{ \pm} \chi .
$$

It is easy to see that $K_{1}$ corresponds to the logarithmically divergent boundary counterterm in the standard SF [9]. It leads to a multiplicative renormalization of the quark and anti-quark boundary fields. In order to understand the rôle of $K_{ \pm}$it is instructive to rotate back to the standard SF. In terms of the primed fields one then obtains

$$
K_{ \pm} \rightarrow-\bar{\chi}^{\prime} i \gamma_{5} \tau^{3} P_{ \pm} \chi^{\prime}
$$

We note that $K_{+}\left(K_{-}\right)$contains only Dirichlet components at $x_{0}=0\left(x_{0}=T\right)$. This means that it will never contribute to the SF correlation functions used in practice. We are thus left with a single counterterm $K_{-}$at $x_{0}=0\left(K_{+}\right.$at $\left.x_{0}=T\right)$, which is composed of non-Dirichlet components only. As it violates parity and flavour symmetries it is a finite counterterm which can be fixed by requiring parity restoration. The extension of this analysis to dimension 4 boundary counterterms will be discussed elsewhere [10].

\section{5. $\mathbf{O}(a)$ improvement and SF boundary conditions}

Consider first massless lattice QCD on a hyper-torus with some variant of periodic boundary conditions for all fields. The cutoff dependence of renormalized correlation functions is then described by Symanzik's effective continuum theory (see [11] for notation and references),

$$
\begin{aligned}
& S_{\text {eff }}=S_{0}+a S_{1}+O\left(a^{2}\right), \\
& \langle O\rangle=\langle O\rangle^{\text {cont }}+a\left\langle S_{1} O\right\rangle^{\text {cont }}+a\langle\delta O\rangle^{\mathrm{cont}}+O\left(a^{2}\right) .
\end{aligned}
$$


Here, $S_{1}$ and $\delta O$ are $\mathrm{O}(a)$ counterterms for the action and for $O$. Chiral symmetry of the continuum action $S_{0}$ implies that all insertions of $\mathrm{O}(a)$ counterterms vanish. To see this more explicitly consider a $\gamma_{5}$ field transformation:

$$
\chi \rightarrow \gamma_{5} \chi, \quad \bar{\chi} \rightarrow-\bar{\chi} \gamma_{5} .
$$

The massless continuum action $S_{0}$ is invariant while $S_{1}$ changes its sign. Assuming that $O$ is even under a $\gamma_{5}$ transformation, one can also show that $\delta O$ has to be odd. It then follows that

$$
\left\langle O S_{1}\right\rangle^{\text {cont }}=-\left\langle O S_{1}\right\rangle^{\text {cont }}, \quad\langle\delta O\rangle^{\text {cont }}=-\langle\delta O\rangle^{\text {cont }}
$$

implying that indeed both counterterm insertions vanish. One may wonder whether the $\mathrm{O}(a)$ ambiguity of the chiral limit might affect this conclusion. This is not the case, as the ambiguity is proportional to

$$
\int \mathrm{d}^{4} x\langle O \bar{\chi}(x) \chi(x)\rangle^{\mathrm{cont}}=0,
$$

which vanishes again due to chiral symmetry. It follows that $\mathrm{O}(a)$ improvement of massless Wilson quarks in finite volume is automatically satisfied (cp. [6]).

The situation changes in the presence of SF boundary conditions. The $\gamma_{5}$ transformation is then no longer a symmetry of the effective continuum theory, as the transformed fields satisfy boundary conditions with the complementary projectors. Indexing correlation functions with the projectors of the boundary condition for $\chi$ at $x_{0}$, one finds for $\gamma_{5}$-even observables $O$,

$$
\langle O\rangle_{\left(P_{ \pm}\right)}^{\text {cont }}=\langle O\rangle_{\left(P_{\mp}\right)}^{\text {cont }}, \quad\left\langle O S_{1}\right\rangle_{\left(P_{ \pm}\right)}^{\text {cont }}=-\left\langle O S_{1}\right\rangle_{\left(P_{\mp}\right)}^{\text {cont }} \neq 0,
$$

i.e. the insertion of $S_{1}$ does not vanish. This should not come as a surprise, as otherwise the determination of $\mathrm{O}(a)$ improvement coefficients such as $c_{\mathrm{sw}}$ or $c_{\mathrm{A}}$ in ref. [12] would have been impossible.

The question then arises whether automatic $\mathrm{O}(a)$ improvement can be achieved with the chirally rotated boundary conditions ${ }^{2}$. This is indeed the case: if one augments the $\gamma_{5}$ transformation by a flavour exchange, i.e.

$$
\chi \rightarrow \gamma_{5} \tau^{1} \chi, \quad \bar{\chi} \rightarrow-\bar{\chi} \gamma_{5} \tau^{1}
$$

the chirally rotated SF boundary conditions remain unchanged, due to $\gamma_{5} \tau^{1} Q_{ \pm}=Q_{ \pm} \gamma_{5} \tau^{1}$. Therefore, for $\gamma_{5} \tau^{1}$-even observables the previous argument holds. Note, however, that this discussion does not account for the $\mathrm{O}(a)$ counterterms at the time boundaries. Some of them share symmetries with the continuum action $S_{0}$ and their insertion therefore never cancels. However, $\mathrm{O}(a)$ improvement may then still be achieved by tuning just a few $\mathrm{O}(a)$ boundary counterterms.

The SF coupling [14] is an example of a $\gamma_{5}$-even observable. In perturbation theory one finds

$$
\bar{g}^{2}(L)=g_{\overline{\mathrm{MS}}}^{2}(\mu)+k_{1}(\mu L) g_{\overline{\mathrm{MS}}}^{4}(\mu)+O\left(g^{6}\right) .
$$

Setting $\mu=L^{-1}$ the fermionic contribution to $k_{1}=k_{1,0}+N_{\mathrm{f}} k_{1,1}$ has been computed in [15],

$$
k_{1,1}=-0.039863(2) /(4 \pi)
$$

\footnotetext{
${ }^{2}$ For a different attempt see [13].
} 
and is obtained as the leading term in the asymptotic behaviour of a series of lattice approximants,

$$
f(L / a) \sim r_{0}+(a / L)\left[r_{1}+s_{1} \ln (a / L)\right]+O\left(a^{2}\right) .
$$

In the standard SF one finds $r_{0}=k_{1,1}$ and $s_{1} \propto c_{\mathrm{Sw}}^{(0)}-1$. Using instead the chirally rotated SF, $k_{1,1}$ is indeed reproduced, while $s_{1}$ now vanishes independently of $c_{\mathrm{sw}}^{(0)}$, as expected.

\section{Acknowledgments}

I would like to thank Margarita García Pérez and Rainer Sommer for useful discussions. Partial support by the Spanish government through a Ramón y Cajal fellowship is gratefully acknowledged.

\section{References}

[1] K. Jansen et al., "Non-perturbative renormalization of lattice QCD at all scales," Phys. Lett. B 372 (1996) 275 [arXiv:hep-lat/9512009].

[2] S. Sint, "Non-perturbative renormalization in lattice field theory," Nucl. Phys. Proc. Suppl. 94 (2001) 79 [arXiv:hep-lat/0011081].

[3] M. Guagnelli, J. Heitger, C. Pena, S. Sint and A. Vladikas [ALPHA Collaboration], "Non-perturbative renormalization of left-left four-fermion operators in quenched lattice QCD," arXiv:hep-lat/0505002.

[4] R. Frezzotti, P. A. Grassi, S. Sint and P. Weisz [Alpha collaboration], "Lattice QCD with a chirally twisted mass term," JHEP 0108 (2001) 058 [arXiv:hep-lat/0101001].

[5] R. Frezzotti, S. Sint and P. Weisz [ALPHA collaboration], "O(a) improved twisted mass lattice QCD," JHEP 0107 (2001) 048 [arXiv:hep-lat/0104014].

[6] R. Frezzotti and G. C. Rossi, "Chirally improving Wilson fermions. I: O(a) improvement," JHEP 0408 (2004) 007 [arXiv:hep-lat/0306014].

[7] S. Sint, "On the Schrödinger functional in QCD," Nucl. Phys. B 421 (1994) 135 [arXiv:hep-lat/9312079].

[8] Y. Taniguchi, "Schrödinger functional formalism with Ginsparg-Wilson fermion," arXiv:hep-lat/0412024.

[9] S. Sint, "One loop renormalization of the QCD Schrödinger functional," Nucl. Phys. B 451 (1995) 416 [arXiv:hep-lat/9504005].

[10] S. Sint, in preparation

[11] M. Lüscher, S. Sint, R. Sommer and P. Weisz, "Chiral symmetry and O(a) improvement in lattice QCD,” Nucl. Phys. B 478 (1996) 365 [arXiv:hep-lat/9605038].

[12] M. Lüscher, S. Sint, R. Sommer, P. Weisz and U. Wolff, "Non-perturbative O(a) improvement of lattice QCD,” Nucl. Phys. B 491 (1997) 323 [arXiv:hep-lat/9609035].

[13] R. Frezzotti and G. Rossi, "Chirally improving Wilson fermions. III: The Schrödinger functional," arXiv:hep-lat/0507030.

[14] M. Lüscher, R. Narayanan, P. Weisz and U. Wolff, "The Schrödinger functional: A renormalizable probe for non-Abelian gauge theories," Nucl. Phys. B 384, 168 (1992) [arXiv:hep-lat/9207009].

[15] S. Sint and R. Sommer, "The Running coupling from the QCD Schrödinger functional: A One loop analysis," Nucl. Phys. B 465 (1996) 71 [arXiv:hep-lat/9508012]. 